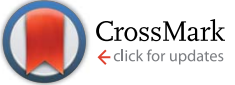

Cite this: RSC Adv., 2017, 7, 13138

\title{
Polyethyleneimine-mediated seed growth approach for synthesis of silver-shell silica-core nanocomposites and their application as a versatile SERS platform $\dagger$
}

\author{
Chongwen Wang, $\}^{\mathrm{ab}}$ Min Li,,$^{\mathrm{bc}}$ Qingjun Li,,$^{\mathrm{bc}}$ Kehan Zhang, ${ }^{\mathrm{b}}$ Chaoguang Wang, ${ }^{\mathrm{b}}$ \\ Rui Xiao*b and Shengqi Wang*abc
}

\begin{abstract}
We synthesized silver-shell silica-core nanoparticles $\left(\mathrm{SiO}_{2} @ \mathrm{aPEI}(\mathrm{Ag} N \mathrm{NPs})\right.$ with complete silver shells through the proposed polyethyleneimine (PEI)-mediated seed growth method. Cationic PEI rapidly selfassembled on the silica spheres through sonication to form a thin multifunctional interlayer, which can adsorb Au seeds densely and uniformly and stabilize the entire structure. Ag shell formation was completed within 2 min, and generated $\mathrm{SiO}_{2} @ \mathrm{PEl} @ \mathrm{Ag} \mathrm{NPs}$ were highly uniform in size and shape with nanoscale roughness. The improved seed growth method can be generally used to coat $\mathrm{Ag}$ shells of different thicknesses on the $\mathrm{SiO}_{2}$ cores with any particle size to form a well-dispersed core/shell nanostructure with high SERS activity. $\mathrm{SiO}_{2} @ \mathrm{CPEI} @ \mathrm{Ag} \mathrm{NPs}$ of different sizes could be versatile SERS platforms for different applications. The potential of these particles as SERS substrates was verified by detection of the pesticide thiram and potential as SERS tags was verified by detection of human IgG, with detection limits as low as $10^{-9} \mathrm{M}$ and $10 \mathrm{pg} \mathrm{mL}^{-1}$, respectively. Hence, $\mathrm{SiO}_{2} @ \mathrm{PPE} @ \mathrm{AAg} \mathrm{NPs}$ are powerful tools for practical SERS detection.
\end{abstract}

Received 23rd December 2016 Accepted 21st February 2017

DOI: 10.1039/c6ra28629a

rsc.li/rsc-advances
Over the past years, scholars have developed various methods for the synthesis of $\mathrm{SiO}_{2} @ A \mathrm{Ag}$ NPs with controllable morphology; these methods include electroless plating, ${ }^{9}$ layer by layer (LBL), ${ }^{10}$ direct deposition, ${ }^{11}$ and seed growth-mediated methods. ${ }^{12}$ The $\mathrm{SnCl}_{2}$-mediated electroless plating method, which was proposed by Liz-Marzán et al., can be used to homogeneously deposit Ag NPs on the $\mathrm{SiO}_{2}$ core but requires repeated adsorption of $\mathrm{Sn}^{2+}$ and $\mathrm{Ag}^{+}$ions onto the surface of the silica particles. ${ }^{13}$ LBL method readily produces Ag nanoshells by stacking Ag NPs on silica core surfaces through electrostatic interaction; this method is time consuming because of multistep deposition and difficulty in producing complete Ag shells. ${ }^{14}$ Several attempts have been made to directly deposit Ag NPs on $\mathrm{SiO}_{2}$ particles by soaking them in ethanolic solutions of $\mathrm{AgNO}_{3}$ and butylamine; ${ }^{15}$ the results are unsatisfactory, that is, complete and controllable thickness $\mathrm{Ag}$ shells were not produced on the core surface. Of these methods, seed-mediated growth method is the most promising approach to grow uniform and complete Ag nanoshells by adding new atoms onto the existing nuclei. ${ }^{16}$ Therefore, additional presynthetic steps are required for absorption or deposition of metal seeds on the silica core surface before subsequent growth of Ag shells. To date, amine-functionalized silica particles have been widely investigated because amine groups act as attachment points for small Au or Ag seeds; silane coupling agents are commonly used for synthesis of amino-modified silica spheres. ${ }^{17,18}$ However, full surface amino modification is sometimes difficult to achieve;

\footnotetext{
${ }^{a}$ College of Life Sciences \& Bio-Engineering, Beijing University of Technology, Beijing 100124, PR China. E-mail: sqwang@bmi.ac.cn

${ }^{b}$ Beijing Institute of Radiation Medicine, Beijing 100850, PR China. E-mail: ruixiao203@sina.com

'Henan University of Chinese Medicine, Zhengzhou, Henan 450008, PR China

$\dagger$ Electronic supplementary information (ESI) available. See DOI: 10.1039/c6ra28629a

$\ddagger$ These authors contributed equally to this work.
} 
thus uniform and dense seed absorption on the core particles cannot be achieved, eventually affecting the complete Ag shell formation.

Basing on our previous studies, ${ }^{19-21}$ we proposed a polyethyleneimine (PEI)-mediated seed growth method to achieve highly reproducible and controllable coating silica spheres with complete $\mathrm{Ag}$ shells in this study. PEI was used to form a bifunctional thin interlayer to achieve the absorption of dense $\mathrm{Au}$ NPs as seeds and keep the nanostructure stable during shell growth. The entire reaction process for Ag shell formation was completed within $2 \mathrm{~min}$; the resulting $\mathrm{SiO}_{2} @ P E I @ A g$ NPs were highly uniform in size and shape with high SERS activity. The SERS activities of the as-prepared $\mathrm{SiO}_{2} @ P E I @ A g$ NPs were tested using $p$-aminothiophenol (PATP) as probe molecule. Moreover, $\mathrm{Ag}$ shells of different thicknesses easily formed outside the silica cores of different sizes depending on different detection demands. On one hand, high-sensitive detection of the pesticide thiram was achieved using large-sized $\mathrm{SiO}_{2}$ @PEI@Ag NPs (300 nm core) as SERS substrates at a detection limit of $10^{-9} \mathrm{M}$, which is lower than the standard safety value (7 $\mathrm{ppm}$ ) prescribed by the U.S. Environmental Protection Agency (EPA).. ${ }^{22}$ On the other hand, small-sized $\mathrm{SiO}_{2} @ P E I @ A g$ NPs ( $80 \mathrm{~nm}$ core) as high-quality SERS tags were successfully applied to SERS-based immunoassay for human immunoglobulin (IgG) detection with a low detection limit of $10 \mathrm{pg} \mathrm{mL}^{-1}$. Hence, $\mathrm{SiO}_{2} @ P E I @ A g$ NPs can be potentially used as effective and versatile SERS tools in practical applications, including pollutant detection, food safety, and immunodetection.

\section{Methods}

\section{Materials and chemicals}

Silver nitrate $\left(\mathrm{AgNO}_{3}\right)$, chlorauric acid tetrahydrate $\left(\mathrm{HAuCl}_{4}-\right.$ $\cdot 4 \mathrm{H}_{2} \mathrm{O}$ ), ammonia solution (28\%), and formaldehyde $(37 \%)$ were purchased from Sinopharm Chemical Reagent Co. Branched PEI (MW 25 000), tetraethoxysylane (TEOS), polyvinylpyrolidone (PVP, MW 40 000), aminothiophenol PATP, 2nitrobenzoic acid (DTNB), 11-mercaptoundecanoic acid (MUA), $N$-(3-dimethylaminopropyl)- $N N^{\prime}$-ethylcarbodiimide hydrochloride (EDC), $N$-hydroxysuccinimide (NHS), thiram, human IgG, and goat anti-human IgG were purchased from Sigma-Aldrich. All other chemicals were purchased from Shanghai Chemical Reagent Co., Ltd. Ultrapure water with resistivity of $18.2 \mathrm{M} \Omega$ $\mathrm{cm}^{-1}$ was used to prepare aqueous solutions.

\section{Preparation of high-performance $\mathrm{SiO}_{2} @ P E I @ A g$ NPs}

$\mathrm{SiO}_{2}$ NPs were prepared according to a modified Stöber method, which involves the ammonia-catalyzed hydrolysis and condensation of TEOS in a mixture of ethanol and water. ${ }^{17}$ The size of $\mathrm{SiO}_{2}$ NPs can be controlled by modulating the molar ratio of TEOS, water, and ammonia. The $\mathrm{SiO}_{2} @$ PEI NPs were synthesized through a PEI self-assembly process under sonication condition. First, $0.5 \mathrm{~g}$ PEI was dissolved in $100 \mathrm{~mL}$ of deionized water by ultrasonication for $10 \mathrm{~min}$. Next, $0.1 \mathrm{~mL}$ of prepared $\mathrm{SiO}_{2} \mathrm{NPs}$ $\left(10 \mathrm{mg} \mathrm{mL}^{-1}\right)$ was dispersed in the PEI solution under sonication at room temperature for $1 \mathrm{~h}$, during which PEI gradually self- assembled on the silica cores. Then, the obtained $\mathrm{SiO}_{2} @$ PEI NPs were centrifuged and rinsed for five times with deionized water to remove the excess PEI. Colloidal 3-5 nm Au NPs were prepared according to the method of YouXing Fang. ${ }^{23}$ After preparation, PEI-modified $\mathrm{SiO}_{2}$ NPs were mixed with 3-5 nm Au NPs and sonicated for $30 \mathrm{~min}$ to form $\mathrm{SiO}_{2} @$ @PEI-Au seed NPs. Briefly, $5 \mathrm{mg}$ of $\mathrm{SiO}_{2} @$ PEI-Au seed was dispersed in $200 \mathrm{~mL}$ of $0.2 \mathrm{mM}$ silver nitrate aqueous solution containing $0.2 \mathrm{wt} \%$ PVP. The mixture was added with excess amounts of $37 \%$ formaldehyde $(150 \mu \mathrm{L})$ and $28 \%$ ammonia solution $(300 \mu \mathrm{L})$ in sequence. The $\mathrm{SiO}_{2} @ P E I @ A g$ core-shell NPs were obtained within 2 min under sonication at $30^{\circ} \mathrm{C}$. The products were centrifuged and redispersed with deionized water to $1 \mathrm{mg} \mathrm{mL} \mathrm{m}^{-1}$.

\section{SERS detection of thiram}

In a typical experiment, a standard thiram solution from $10^{-5} \mathrm{M}$ to $10^{-10} \mathrm{M}$ was prepared using a thiram stock solution. For the SERS measurement, $500 \mu \mathrm{L}$ of thiram solution of different concentrations was mixed with $50 \mu \mathrm{L}$ of $\mathrm{SiO}_{2} @ P E I @ A g$ NPs (300 $\mathrm{nm}$ core) in $1.5 \mathrm{~mL}$ centrifuge tube, and the mixture was incubated under sonication for $1 \mathrm{~h}$. Then, $\mathrm{SiO}_{2} @ P E I @ A g$ NPs was separated by centrifugation and rinsed twice with ethanol to remove excess thiram. After washing, the precipitate was resuspended in $5 \mu \mathrm{L}$ of ethanol. The suspension was dropped on a silicon substrate and air dried. The SERS spectra of the sample were then recorded.

\section{Preparation of $\mathrm{SiO}_{2} @ P E I @ A g$ NPs SERS tags for the SERS immunoassay}

Briefly, $100 \mu \mathrm{L}$ of $10 \mathrm{mM}$ DTNB was added to $10 \mathrm{~mL}$ of the asprepared $\mathrm{SiO}_{2} @$ PEI@Ag NPs $(80 \mathrm{~nm})$ solution. The mixture was vigorously stirred at $30{ }^{\circ} \mathrm{C}$ for $2 \mathrm{~h}$. After purification (5000 rpm for $5 \mathrm{~min}$ ), the $\mathrm{SiO}_{2} @ P E I @ A g / D T N B$ was linked with goat anti-human IgG $\left(10 \mu \mathrm{L}, 10 \mathrm{mg} \mathrm{mL}^{-1}\right)$ by covalent bonding through the EDC/NHS chemistry. For blocking the unreacted carboxyl sites on the $\mathrm{SiO}_{2}$ @PEI@Ag surface, $100 \mu \mathrm{L}$ of BSA $(10 \%, \mathrm{v} / \mathrm{v})$ was added to the mixture and allowed to react for an additional $1 \mathrm{~h}$. To remove any excess unbound antibody, the solution was centrifuged at $4000 \mathrm{rpm}$ for three times. Finally, $\mathrm{SiO}_{2} @ P E I @ A g$ SERS tags were obtained by re-dispersing the precipitates into $5 \mathrm{~mL}$ of phosphate-buffered saline (PBS, $10 \mathrm{mM}, \mathrm{pH}$ 7.4) and stored at $4{ }^{\circ} \mathrm{C}$ before use.

\section{Preparation of immunomagnetic beads}

Magnetic microspheres $\left(\mathrm{a}-\mathrm{Fe}_{2} \mathrm{O}_{3}, 1.5 \mu \mathrm{m}\right)$ were synthesized following the method proposed by Gan et al. ${ }^{24}$ Briefly, $100 \mathrm{mg}$ of the as-prepared $\mathrm{Fe}_{2} \mathrm{O}_{3}$ particles were dispersed in a deionized water-ammonia-ethanol mixture $(10 \mathrm{~mL} / 8 \mathrm{~mL} / 180 \mathrm{~mL})$ by sonication for $10 \mathrm{~min}$ to prepare $\mathrm{a}-\mathrm{Fe}_{2} \mathrm{O}_{3} @ \mathrm{SiO}_{2}$ microspheres. Subsequently, $100 \mu \mathrm{L}$ of TEOS in ethanol was gradually dropped into the solution by vigorous sonication at room temperature for 60 min. The obtained a- $\mathrm{Fe}_{2} \mathrm{O}_{3} @ \mathrm{SiO}_{2}$ microspheres were collected using a magnet, followed by washing with deionized water and ethanol for three times each.

For preparation of immunomagnetic beads, a- $\mathrm{Fe}_{2} \mathrm{O}_{3} @ \mathrm{SiO}_{2}$ microspheres were carboxyl group-functionalized and 
conjugated with antibody according to a previous method reported by $\mathrm{Li}$ et al. ${ }^{25,26}$ Briefly, $100 \mu \mathrm{L}$ of triethoxysilylpropyl succinic anhydride (TEPSA) was added to $10 \mathrm{~mL}$ of the a-Fe ${ }_{2}$ $\mathrm{O}_{3} @ \mathrm{SiO}_{2}$ microsphere aqueous solution and incubated overnight. Carboxyl-terminated $\mathrm{a}-\mathrm{Fe}_{2} \mathrm{O}_{3} @ \mathrm{SiO}_{2}$ microspheres were obtained after magnetic separation and redispersed in $1 \mathrm{~mL}$ of PBS solution (10 mM, pH 7.4). Subsequently, EDC (100 $\mu \mathrm{L}, 10$ $\mathrm{mM})$ and NHS $(20 \mu \mathrm{L}, 100 \mathrm{mM})$ were added to $100 \mu \mathrm{L}$ of the carboxylated a- $\mathrm{Fe}_{2} \mathrm{O}_{3} @ \mathrm{SiO}_{2}$ microspheres $\left(5 \mathrm{mg} \mathrm{mL}^{-1}\right)$ solution. The mixture was added with PBS buffer (10 mM, pH 7.4) until the total volume reached $1 \mathrm{~mL}$. The mixture was shaken for $20 \mathrm{~min}\left(800 \mathrm{rpm}, 37^{\circ} \mathrm{C}\right)$, added with $5 \mu \mathrm{L}$ of goat anti-human IgG (10 mg mL ${ }^{-1}$ in PBS), and shaken for another $1 \mathrm{~h}$. The products were magnetically separated and rinsed three times with PBS (10 mM, pH 7.4) to remove excess antibodies. The antibody-conjugated a- $\mathrm{Fe}_{2} \mathrm{O}_{3} @ \mathrm{SiO}_{2}$ microspheres were blocked with $100 \mu \mathrm{L}$ of BSA $(10 \%, v / v)$ for $1 \mathrm{~h}$ under the same shaking condition to avoid nonspecific absorption. The resulting immunomagnetic beads were stored at $4{ }^{\circ} \mathrm{C}$ before use.

\section{Immunoassay protocol}

In a typical experiment, $500 \mu \mathrm{L}$ of PBS buffer containing the target human IgG at various concentrations was prepared and incubated with $10 \mu \mathrm{L}$ of the capture antibody-conjugated immunomagnetic beads for $30 \mathrm{~min}$ at $37^{\circ} \mathrm{C}$ with gentle shaking. After immunocapture of the target human IgG, the immune complexes were magnetically collected in the mixture by applying an external magnet and washed twice with PBS containing Tween-20 buffer (PBST, 0.05\%, v/v). Subsequently, $200 \mu \mathrm{L}$ of the $\mathrm{SiO}_{2} @ P E I @ A g$ SERS tags were added and incubated for another $45 \mathrm{~min}$ at $37{ }^{\circ} \mathrm{C}$ with gentle shaking. The resulting sandwich immune complexes were washed three times with PBST $(0.05 \%, \mathrm{v} / \mathrm{v})$, resuspended in $2 \mu \mathrm{L}$ of deionized water, and transferred to a silicon substrate for SERS measurements.

\section{Instruments}

Transmission electron microscopy (TEM) images were recorded on a Hitachi $\mathrm{H}-7650$ microscope at an accelerating voltage of $200 \mathrm{kV}$. The samples for HRTEM imaging recorded on a Philips Tecnai G2 F20 microscope equipped with energy-dispersive Xray analysis system. Scanning electron microscopy (SEM) analysis was performed with a JEOL JSM-7001F microscopy at an accelerating voltage of $5 \mathrm{kV}$. Elemental mapping images were acquired by energy-dispersive X-ray spectroscopy (EDS) using another JEOL JEM-2100 electron microscope equipped with a STEM unit. Powder X-ray diffraction (XRD) patterns of the products were investigated on a Japan Rigaku D/max $2550 \mathrm{VB} /$ $\mathrm{PC}$ rotation anode X-ray diffractometer. UV-visible spectra were recorded with a Shimadzu 2600 spectrometer. Zeta potential was measured by dynamic light scattering with Zetasizer Nano ZS (Malvern, UK). Raman measurement was
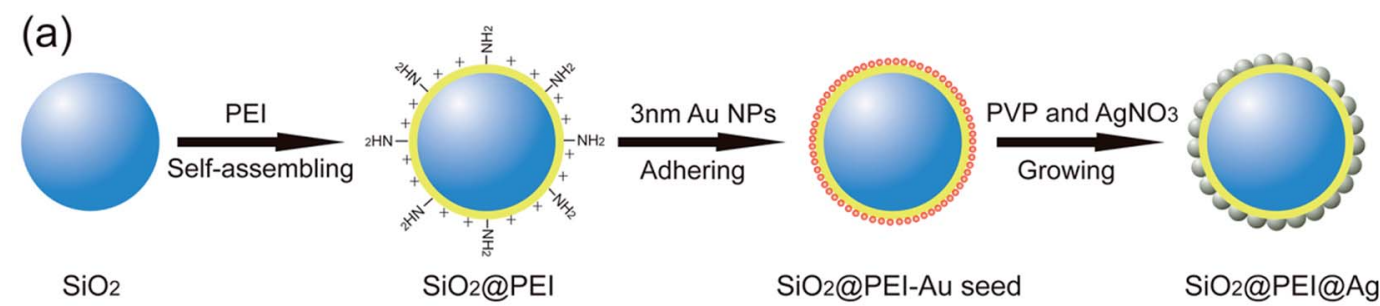

SiO2@PEI-Au seed

SiO2@PEl@Ag
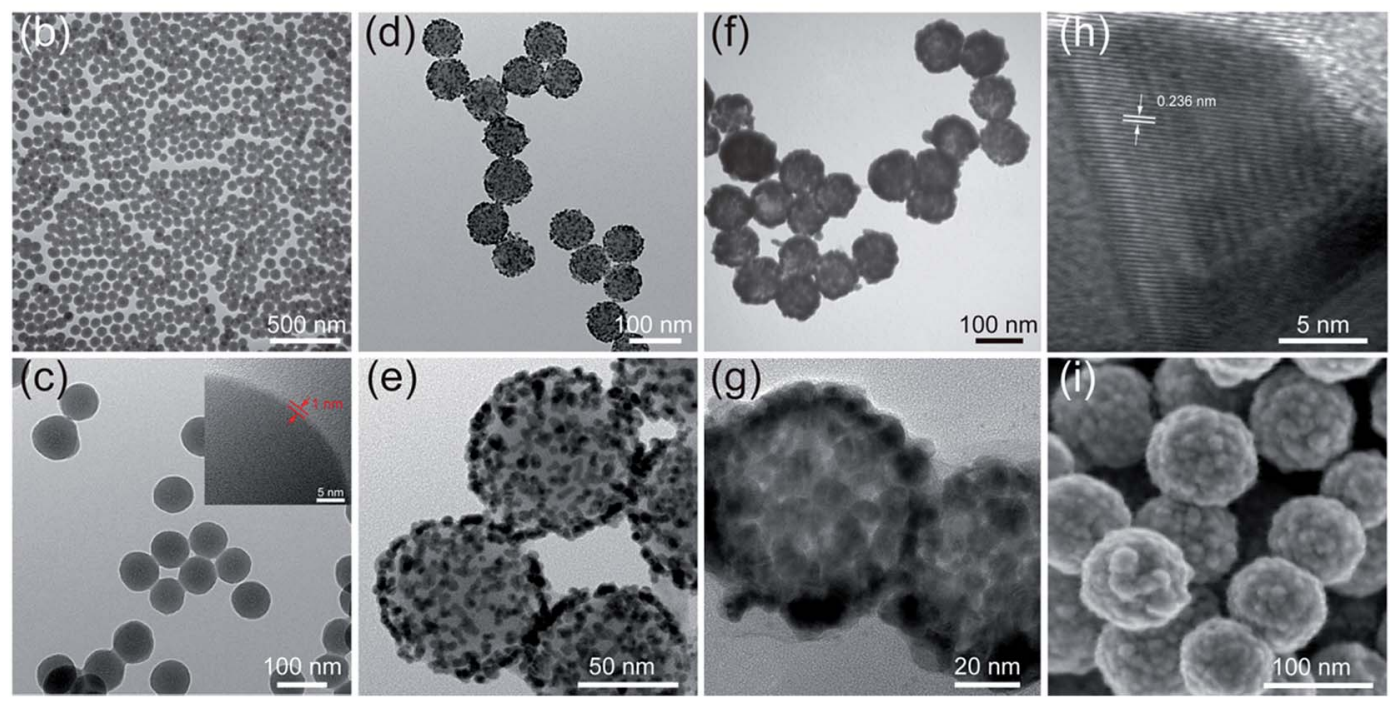

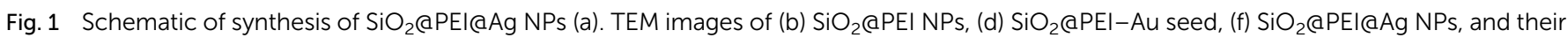
corresponding magnified TEM images in (c), (e), and (g), respectively. (h) HRTEM picture of a part of Ag shell in (g), and (i) SEM image of $\mathrm{SiO}_{2} \mathrm{QPEI}(\mathrm{Agg} N \mathrm{~N}$. The inset in (c) is the corresponding magnified HRTEM image of its edge (arrows indicate a thin PEI shell). 
performed at room temperature on a Renishaw inVia plus Raman system with a $785 \mathrm{~nm}$ excitation wavelength. Incident radiation was coupled into an Olympus BX51 optical microscope and focused to a $2 \mu \mathrm{m}$-diameter spot through a $50 \times$ objective. Data acquisition time was usually $1 \mathrm{~s}$, and the peak intensities of the samples were normalized with respect to the silicon wafer at $520 \mathrm{~cm}^{-1}$.

\section{Results and discussion}

\section{Characterization of $\mathrm{SiO}_{2} @ P E I @ A g$ nanoparticles}

Fig. 1a shows the fabrication of high-performance $\mathrm{SiO}_{2} @$ PEI@Ag NPs. The first step is the formation of the $\mathrm{SiO}_{2} \mathrm{NPs}$ used as cores through Stöber method. Second, $\mathrm{SiO}_{2} @ P E I$ NPs were prepared with the cationic PEI coated on the silica core under sonication. PEI possesses plenty of primary amine groups and positive charges; thus, PEI can assemble quickly on the surface of the negatively charged particles to achieve surface amino group functionalization. Third, dense negatively charged 3-5 nm Au NPs were absorbed on the surface of $\mathrm{SiO}_{2} @ P E I$ NPs as seeds by positive electricity of PEI. These small Au NPs were attached firmly to $\mathrm{SiO}_{2}$ @PEI NPs through sonication because of the covalent binding among the $-\mathrm{NH}_{2}$ groups of the PEI and $\mathrm{Au}$ NPs. Finally, high-performance $\mathrm{SiO}_{2} @ P E I @ A g$ NPs were quickly obtained by adopting our previously reported seed-mediated growth method. ${ }^{19}$ The complete Ag shells were formed within several seconds through the isotropic growth of all Au seeds and the stabilization brought about by PVP.

TEM and SEM analyses were employed to examine the morphology of the as-obtained samples in different stages. Monodispersed $\mathrm{SiO}_{2}$ NPs $(80 \mathrm{~nm})$ were prepared using the modified Stöber method, and the $\mathrm{SiO}_{2}$ NPs were uniform with smooth surface (Fig. S1 $\dagger$ ). The hydrophilic polymer PEI selfassembled easily on the surface of $\mathrm{SiO}_{2}$ NPs by sonication and could not affect the dispersity of $\mathrm{SiO}_{2}$ NPs (Fig. 1b). The HRTEM image confirms the uniform coating of PEI with $1 \mathrm{~nm}$ thickness (Fig. 1c). After the $\mathrm{SiO}_{2} @$ @EI with 3-5 nm Au seed was coated, the TEM image in Fig. 1d shows that the dense small Au NPs spread uniformly on the surface of the $\mathrm{SiO}_{2} @ P E I$ NPs. The Au seeds on the $\mathrm{SiO}_{2}$ NPs served as nucleation sites for subsequent fabrication of the complete Ag shell. Fig. 1f shows the TEM image of the prepared $\mathrm{SiO}_{2} @ \mathrm{PEI} @ \mathrm{Ag} \mathrm{NPs}$, and the size of the particles increased from $80 \mathrm{~nm}$ to approximately $100 \mathrm{~nm}$. The thickness of the Ag shell was approximately $10 \mathrm{~nm}$. A strong contrast difference between the magnified TEM images in Fig. 1e (discontinuous dots) and Fig. 1g (continuous shell) was observed, indicating that the complete $\mathrm{Ag}$ shell was formed successfully on $\mathrm{SiO}_{2}$ NPs. The HRTEM image of a part of the Ag shell is shown in Fig. $1 \mathrm{~h}$; the space between two crystal planes is $0.236 \mathrm{~nm}$, corresponding to the (111) plane of Ag with facecentered cubic (fcc) phase. ${ }^{27}$ To investigate the structure of the shell, we characterized the obtained samples by SEM. The SEM image (Fig. 1i) shows that numerous large Ag NPs covered the entire surface of the nanocomposite, forming a continuous shell with nanoscale roughness.

$\mathrm{SiO}_{2}$ NPs function as supporting core that effectively stabilizes nanocomposites in the solution to prevent aggregation; the as-obtained products at different stages exhibited excellent water dispersibility (Fig. 2a). As shown in Fig. 2b, the $\mathrm{SiO}_{2}$ @PEIAu seed displayed an absorption peak at about $528 \mathrm{~nm}$ (curve c), whereas $\mathrm{SiO}_{2}$ and $\mathrm{SiO}_{2} @$ PEI NPs showed no characteristic absorption peaks (curves a and b); this finding suggests that large amounts of Au NPs were successfully absorbed on the $\mathrm{SiO}_{2}$ NPs. After the Ag shell was formed, an obvious broad plasmonic resonance peak appeared at approximately $384 \mathrm{~nm}$ because of the Mie plasmonic resonance from the Ag NPs (curve d). ${ }^{28}$ The zeta potentials of $\mathrm{SiO}_{2} @ \mathrm{PEI} @ A g$ NPs were measured at different stages of synthesis. Fig. 2c shows that the zeta potentials of the $\mathrm{SiO}_{2} \mathrm{NPs}$ increased from $-35.7 \mathrm{mV}$ to $+45.2 \mathrm{mV}$ when PEI was

(a)

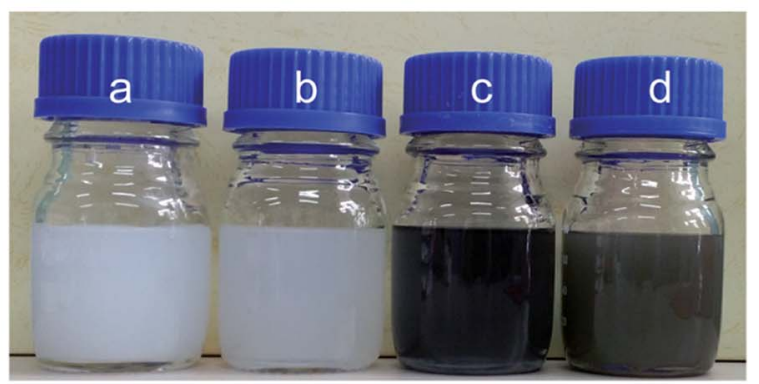

(b)

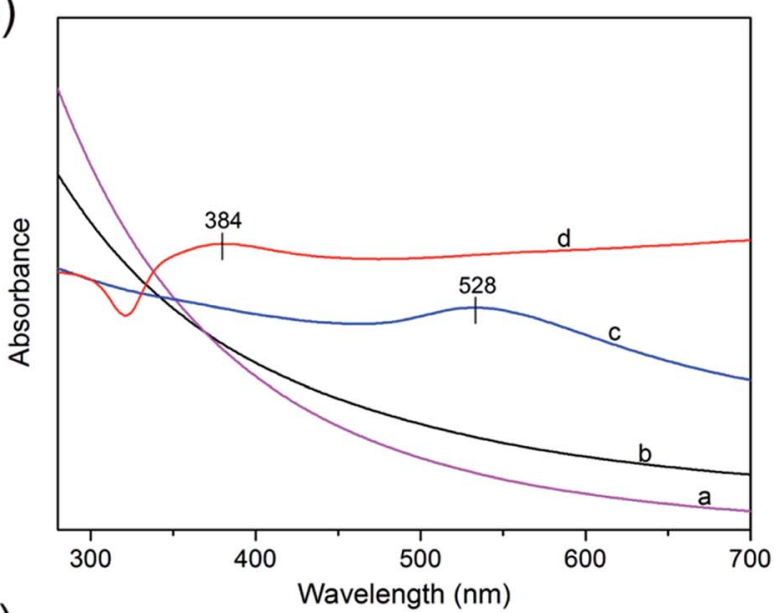

(c)

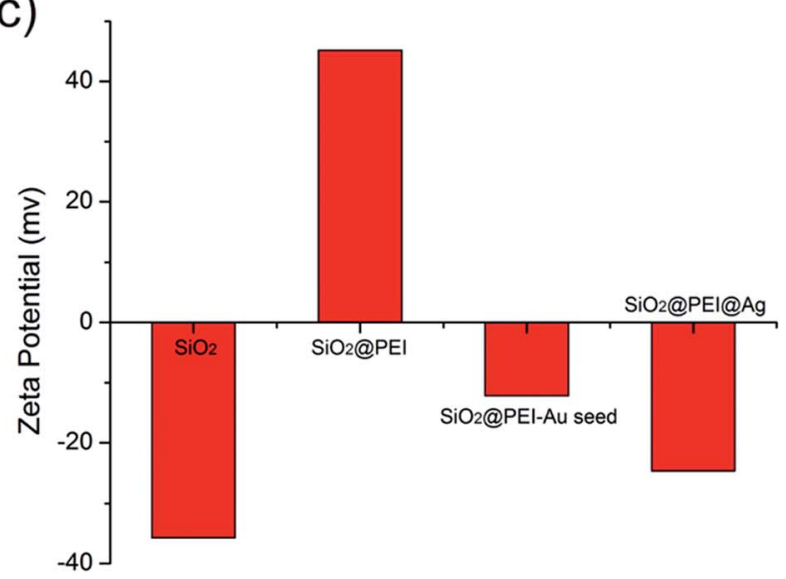

Fig. 2 Photographs (a), UV-vis spectra (b), and statistical results of the zeta potentials (c) of the as-obtained samples during different stages: a- $\mathrm{SiO}_{2}, \mathrm{~b}-\mathrm{SiO}_{2} \mathrm{aPEl}, \mathrm{c}-\mathrm{SiO}_{2} \mathrm{aPEI}-\mathrm{Au}$ seed, and d-SiO${ }_{2} \mathrm{aPEI}(\mathrm{A} \mathrm{Ag} \mathrm{NPs}$. 
(a)

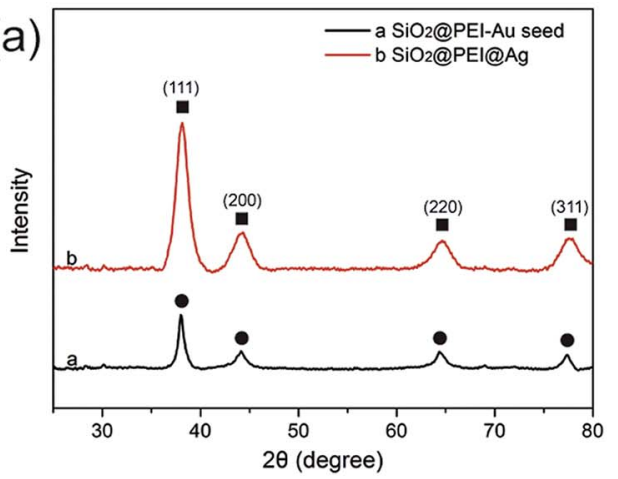

(b)

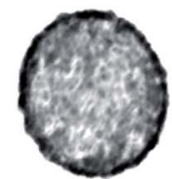

$100 \mathrm{~nm}$
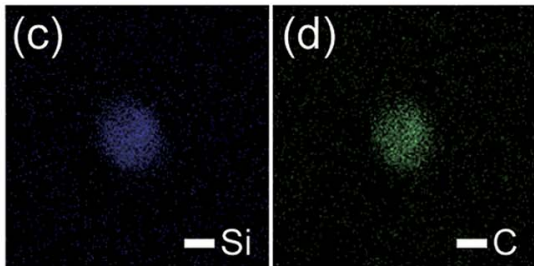

(e)

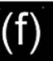

Fig. 3 (a) Typical XRD patterns of the as-synthesized $\mathrm{SiO}_{2} @ \mathrm{aPEI}-\mathrm{Au}$ seed and $\mathrm{SiO}_{2} @ \mathrm{aPEI} @ \mathrm{Ag} \mathrm{NPs}$. The circles label the peaks of cubic Au, whereas the squares label the peaks of cubic Ag. (b) Bright-field TEM image of a single $\mathrm{SiO}_{2} @ \mathrm{aPEI} @ \mathrm{Ag} N \mathrm{NP}$ and (c-f) the associated elemental mappings showing the element distributions of $\mathrm{Si}(\mathrm{c}, \mathrm{blue}), \mathrm{C}$ ( $\mathrm{d}$, green), Au (e, yellow), and Ag ( $\mathrm{f}$, violet) in the nanocomposite.

modified. This change implies that positively charged PEI completely assembled onto the surface of $\mathrm{SiO}_{2}$ particles. After coating of the $\mathrm{Au}$ seeds and the formation of the Ag shell, the zeta potential became negative again. The detailed data of the zeta potentials are shown in Fig. S2. $\dagger$
Powder XRD was applied to evaluate the crystalline properties of the as-prepared samples. As displayed in Fig. 3a, the synthesized $\mathrm{SiO}_{2} @ P E I-A u$ seed exhibited X-ray diffraction peaks, which could be indexed to the cubic structure of the $\mathrm{Au}$ crystals (JCPDS No. 04-0784). ${ }^{29}$ No diffraction peaks

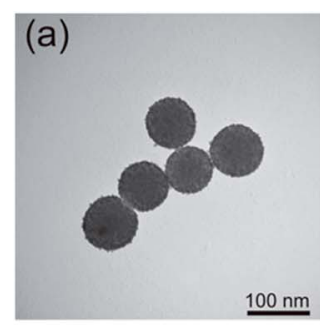

(f)

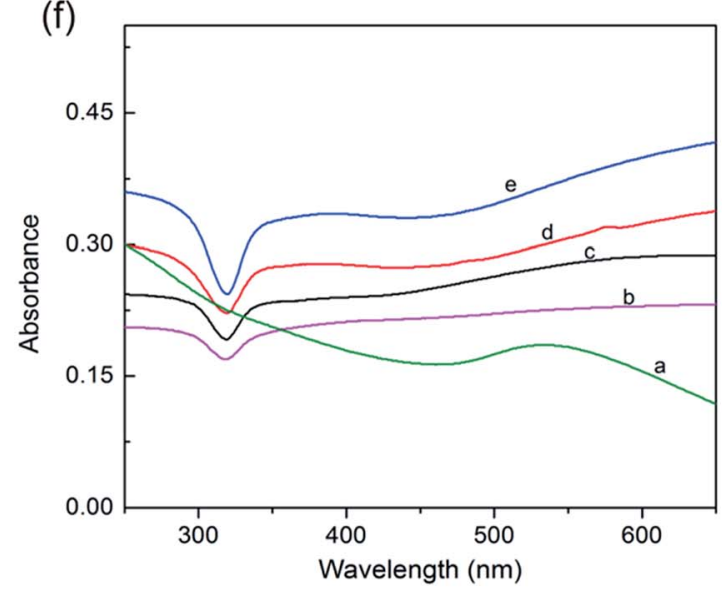

(c)

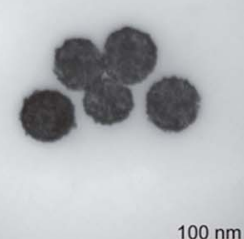

$\underline{100 \mathrm{~nm}}$

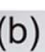

(b)

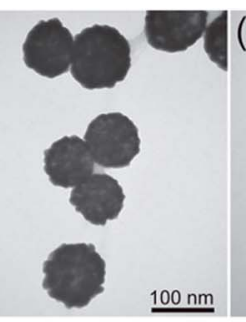

(d)

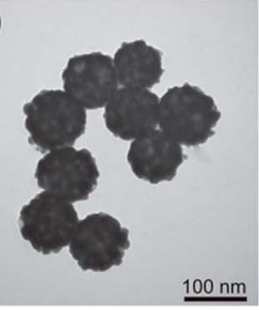

(e)

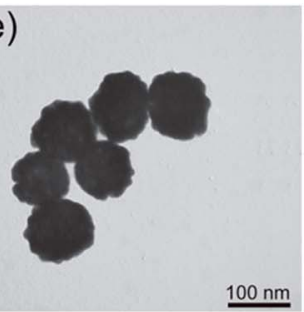

(g)

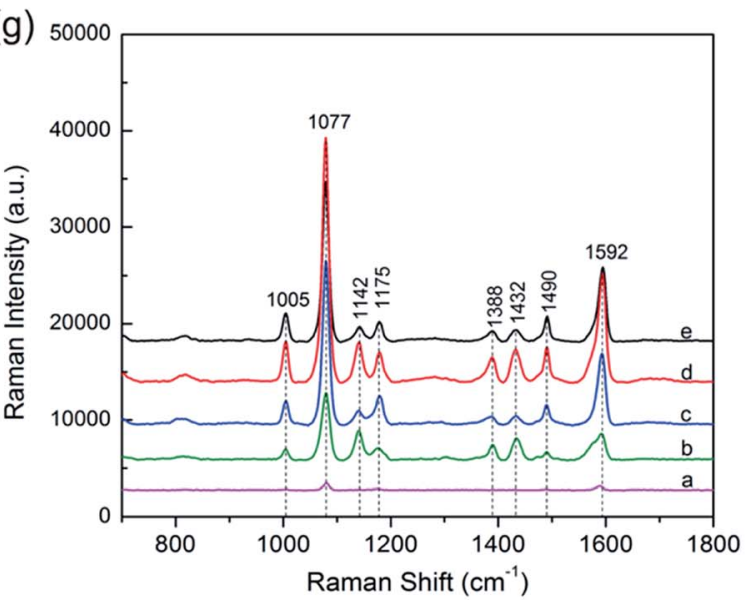

Fig. 4 (a-e) TEM images of $\mathrm{SiO}_{2}$ (aPEl@Ag synthesized with different concentrations of $\mathrm{AgNO}_{3}$. (a) 0, (b) 0.05, (c) 0.1, (d) 0.2, and (e) 0.3 mM. (f) UV-visible spectra of $\mathrm{SiO}_{2}$ (PEl@Ag synthesized with different concentrations of $\mathrm{AgNO}_{3}$ : (a) -0 , (b) $-0.05,(\mathrm{c})-0.1$, (d) -0.2 , and (e) $-0.3 \mathrm{mM}$, and their corresponding Raman spectra of PATP (g). 

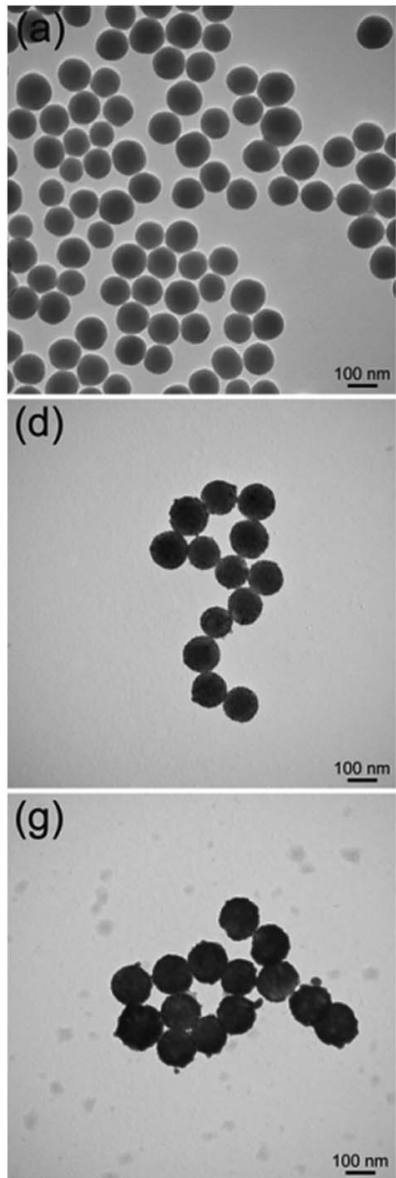

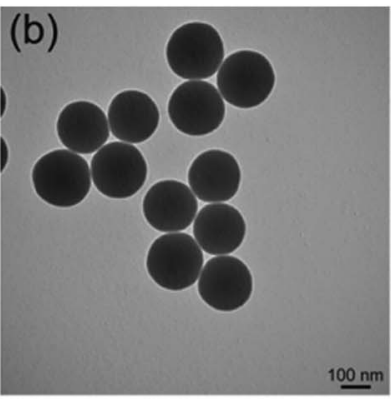

(e)

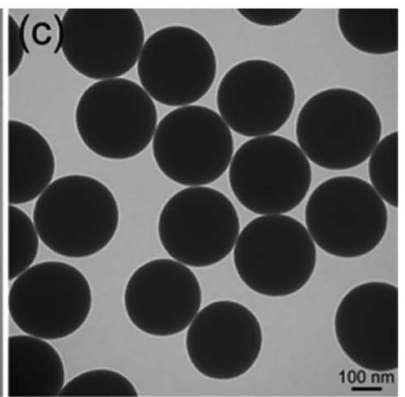

(f)
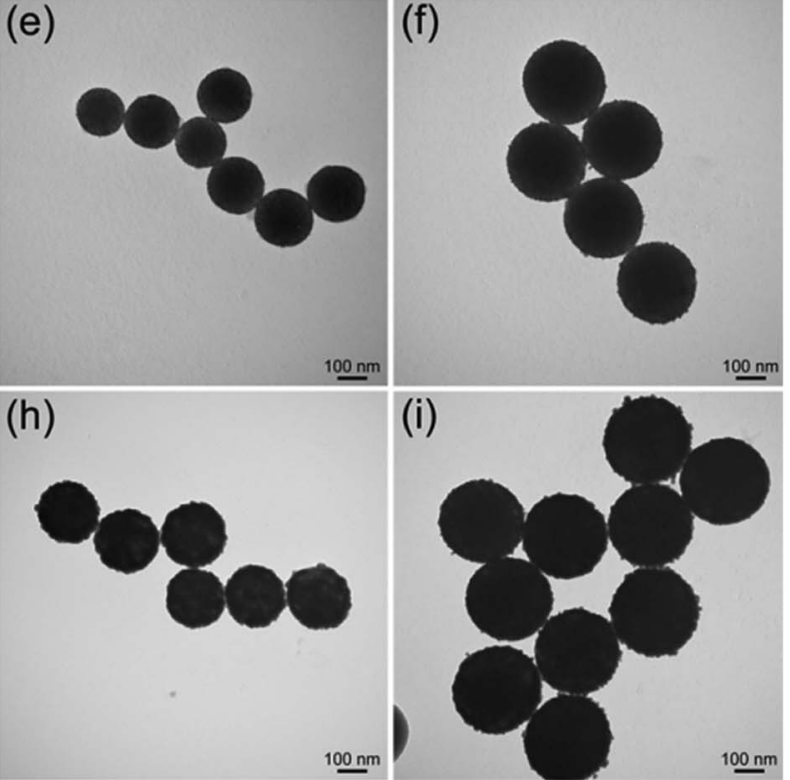

Fig. 5 (a-c) TEM images of $\mathrm{SiO}_{2}$ @PEI NPs with different sizes: (a) 120, (b) 200, and (c) $300 \mathrm{~nm}$, and their corresponding fabricated SiO 2 @PEI-Au seed (d), (e), and (f), and $\mathrm{SiO}_{2} @ \mathrm{aPEl} @ A \mathrm{Ag} N \mathrm{~s}$ in (g), (h), and (i), respectively.

corresponding to $\mathrm{SiO}_{2}$ and PEI were observed because of their amorphous form. Further examination of the $\mathrm{SiO}_{2} @ \mathrm{PEI} @ \mathrm{Ag}$ NPs showed no evident variation in peak positions, but a pronounced increase in intensity was observed when the Ag shells were formed. Four diffraction peaks were observed at $38.1^{\circ}, 44.2^{\circ}, 64.4^{\circ}$, and $77.4^{\circ}$, which correspond to the (111), (200), (220), and (311) planes of Ag with the fcc phase, respectively. ${ }^{30}$ This finding is acceptable because the $\mathrm{Au}$ and $\mathrm{Ag}$ nanocrystals showed minimal differences in the XRD pattern. ${ }^{31}$ $\mathrm{X}$-ray elemental mapping analysis was performed to provide additional insights into the definite morphology and formation mechanism of $\mathrm{SiO}_{2} @ P E I @ A g$ NP synthesis. Fig. 3c-f shows the elemental mapping of a single $\mathrm{SiO}_{2} @ P E I @ A g$ NP. The Si and C elements are distributed throughout the particles, whereas the $\mathrm{Au}$ and Ag signals are mainly enriched on the surface of the nanocomposites.

The strategy of Ag shell formation involves seed-mediated growth. Uniform $\mathrm{Ag}$ shells were produced by a one-step redox reaction in the presence of small and dense Au NPs absorbed on the $\mathrm{SiO}_{2}$ NPs. When ammonia was added, $\mathrm{Ag}^{+}$was reduced by a general reductant $\left(\mathrm{CH}_{2} \mathrm{O}\right)$ within several seconds and was deposited on the Au seeds of $\mathrm{SiO}_{2} @ P E I$, resulting in a discontinuous Ag NPs shell. With increasing $\mathrm{AgNO}_{3}$ concentration, the
Ag NPs gradually became large, finally intersecting with each other and forming a continuous Ag shell. Notably, PVP plays important roles in this process. PVP covers the facets of the $\mathrm{Au}$ seeds; PVP not only prevents the aggregation of nanoparticles but also facilitates the isotropic growth of $\mathrm{Ag}$ particles to form uniform Ag shells.

Based on our proposed PEI-mediated seed growth method, controlling the size and coating condition of $\mathrm{Ag}$ shells can be easily realized by changing the concentration of $\mathrm{AgNO}_{3}$. Fig. 4ae shows the morphologies of the $\mathrm{SiO}_{2} @ P E I @ A g$ NPs synthesized with different concentrations of $\mathrm{AgNO}_{3}$ while the other parameters remained constant. When low concentrations of $\mathrm{AgNO}_{3}$ (such as 0.05 or $0.1 \mathrm{mM}$ ) was added, $\mathrm{Ag}^{+}$was reduced on the surface of the absorbed Au seeds but not in amounts sufficiently large to form a continuous shell Fig. $4 \mathrm{~b}$ and c. When the concentration of $\mathrm{AgNO}_{3}$ was increased to $0.2 \mathrm{mM}$, the resulting Ag shell became slightly rough but basically continuous (Fig. 4d). Furthermore, the Ag shell became absolutely continuous when the $\mathrm{AgNO}_{3}$ concentration reached $0.3 \mathrm{mM}$ (Fig. 4e). These experimental results indicate that the coverage and thickness of the $\mathrm{Ag}$ shells can be well controlled by increasing the concentration of $\mathrm{AgNO}_{3}$ from $0 \mathrm{mM}$ to $0.3 \mathrm{mM}$ while keeping all other parameters fixed. 
(a)

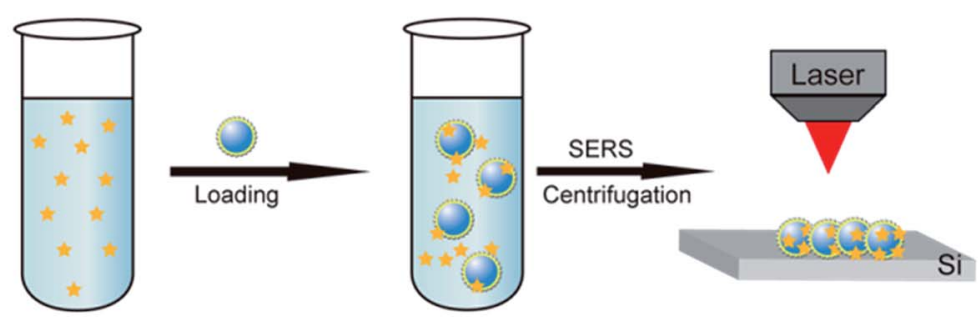

(b)
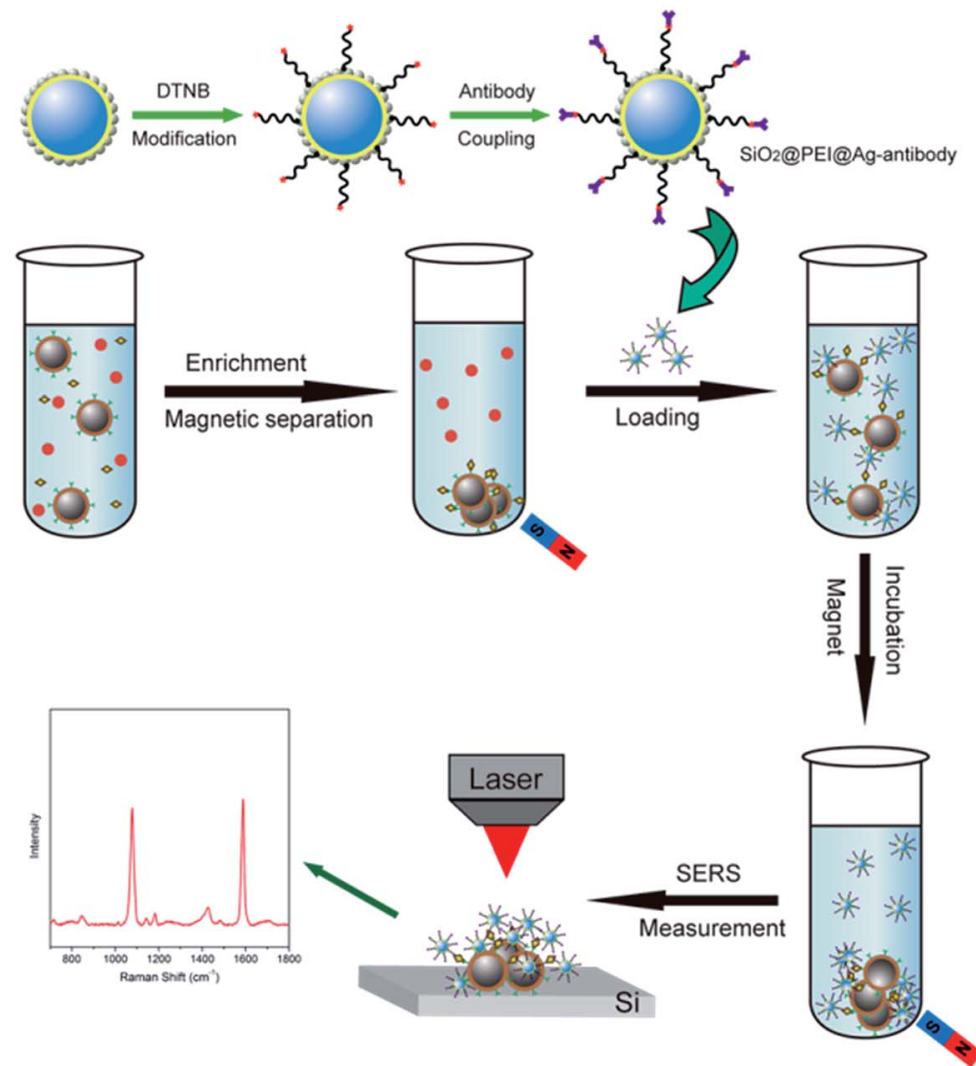

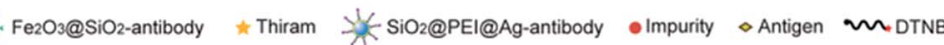

Scheme 1 (a) SERS detection protocol for specific binding molecule (thiram) by using the $\mathrm{SiO}_{2}(\mathrm{aPEl}$ (A) NPs as SERS substrates. (b) Sketch maps of the preparation of $\mathrm{SiO}_{2}$ @PEI@Ag SERS tags and the SERS immune protocol for human IgG detection.

The UV-vis spectra of the $\mathrm{SiO}_{2} @ P E I @ A g$ NPs synthesized with different concentrations of $\mathrm{AgNO}_{3}$ are shown in Fig. 4f. All products were diluted with deionized water for adsorption spectrum measurement. The broad absorption peak of Ag shell was observed from the $\mathrm{SiO}_{2} @ \mathrm{PEI} @ A g$, likely due to the coupling between neighboring $\mathrm{Ag}$ nanoparticles inside the shell region, and large scattering from the $\mathrm{SiO}_{2}$ and $\mathrm{Ag}$ shell. ${ }^{32}$ Similar observation was also reported by Liu and co-workers. ${ }^{12}$ Moreover, as the $\mathrm{Ag}$ shells on the $\mathrm{SiO}_{2}$ cores became continuous and thick, the plasmon resonance peaks slightly red-shifted, and the peak width became narrower. The change in plasmon resonance peak can be explained by plasmon resonance excitation from the close $\mathrm{Ag}$ NPs; this phenomenon indicates that the $\mathrm{Ag}$ shell changed from incomplete to complete.
SERS was first observed in the 1970s on an electrochemically roughened $\mathrm{Ag}$ electrode, which showed an anomalous millionfold enhancement in Raman scattering intensity. ${ }^{33} \mathrm{Ag}$ NPs and $\mathrm{Ag}$ nanoshells enjoy a reputation as an ultrasensitive substrate for SERS. ${ }^{34}$ Considering the Ag shell with nanoscale roughness and controllable morphology, these $\mathrm{SiO}_{2} @ P E I @ A g$ NPs must be efficient SERS substrates under the irradiation of a visible laser. We evaluated the SERS activity of $\mathrm{SiO}_{2} @ P E I @ A g$ NPs by using PATP as the model SERS probe. According to the theory proposed by Wu et al., the PATP molecules adsorbed on the nanoscale Ag surfaces undergo catalytic coupling reaction to selectively produce a new surface species $p, p^{\prime}$-dimercaptoazobenzene (DMAB), an aromatic azo compound. ${ }^{35,36}$ The Raman shifts at 1432 and $1388 \mathrm{~cm}^{-1}$ are related to the $\mathrm{N}=\mathrm{N}$ stretching 

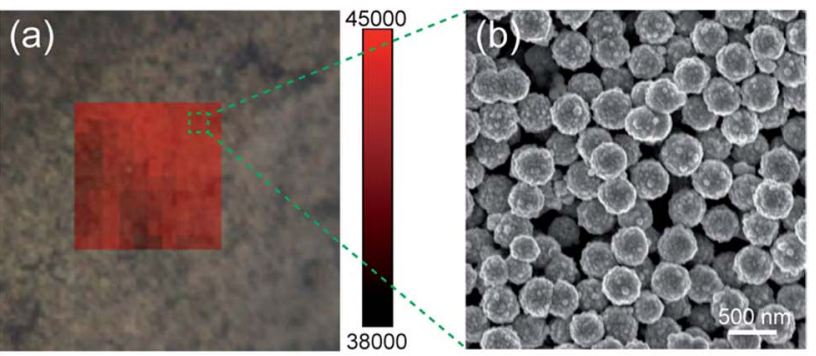

(c)

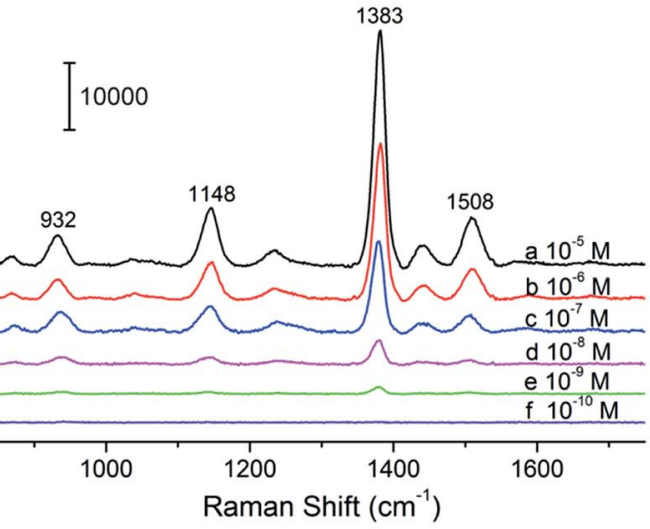

(d)

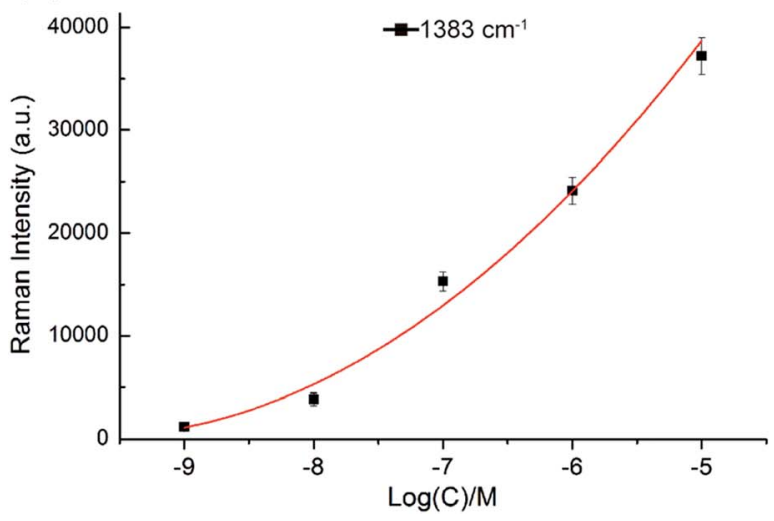

Fig. $6 \mathrm{SiO}_{2}$ (aPEI@Ag NPs (300 nm core) as high-performance SERS substrates for the in situ detection of pesticide thiram. (a) Area mapping of the SERS signal on $\mathrm{SiO}_{2}$ (aPEI@Ag NPs with thiram $\left(10^{-5} \mathrm{M}\right)$. The Raman map was recorded with the Raman peak at $1383 \mathrm{~cm}^{-1}$ and a $1 \mu \mathrm{m}$ step. (b) SEM image of the $\mathrm{SiO}_{2} @$ aPEI@Ag NPs on a Si chip. (c) SERS spectra of thiram with different concentrations on the $\mathrm{SiO}_{2} \mathrm{a}$ PEl@Ag NPs. (d) The intensity-concentration calibration curve for thiram at a concentration range of $1.0 \times 10^{-5}$ to $1.0 \times 10^{-9} \mathrm{M}$ by using SERS intensity at $1383 \mathrm{~cm}^{-1}$. Each spectrum is the average of five independently collected spectra.

vibrations of DMAB, whereas typical SERS peaks of PATP are primarily located at 1592 and $1077 \mathrm{~cm}^{-1}$. As revealed in Fig. 4g, the $\mathrm{SiO}_{2} @ \mathrm{PEI}-\mathrm{Au}$ seed possesses the lowest Raman intensities because of the presence of small Au NPs on the silica surface (curve a). The SERS activity of $\mathrm{SiO}_{2} @ P E I @ A g$ NPs was gradually enhanced as the $\mathrm{AgNO}_{3}$ concentration increased (curves b-d) because the Ag shell thickness and coverage increased through subsequent growth. However, the overgrowth of $\mathrm{Ag}$ shell decreased the SERS activity of $\mathrm{SiO}_{2} @ P E I @ A g$ NPs (curve e) because the nanogaps on the Ag shells disappeared. By contrast, we can conclude that the $\mathrm{SiO}_{2} @ P E I @ A g$ NPs with a nearly complete $\mathrm{Ag}$ shell prepared with $0.2 \mathrm{mM} \mathrm{AgNO}_{3}$ were the most efficient SERS active substrates in series. Furthermore, we examined the detection sensitivity of the $\mathrm{SiO}_{2} @ P E I @ A g$ NPs $\left(0.2 \mathrm{mM} \mathrm{AgNO}_{3}\right)$ for detection of PATP with different concentrations. As shown in Fig. S3a, $\uparrow$ the limit of detection (LOD) for PATP with $\mathrm{SiO}_{2} @ P E I @ A g$ NPs was as low as $1 \times 10^{-10} \mathrm{M}$. The accumulation time was set at $1 \mathrm{~s}$ and the incident power at $1 \%$ of the laser power when recording the Raman spectra. We conducted five tests for each sample, and the results were statistically analyzed to obtain an intensity-concentration calibration curve (polynomial fitting); the curve shows the plot of the intensity of the SERS peak at $1077 \mathrm{~cm}^{-1}$ versus the logarithmic concentration of the sample (Fig. S3bi). Furthermore, the SERS enhancement factors (EF) for PATP were roughly estimated by an adjusted method, as described in detail in ESI S4. $\dagger$ The EF value of the peak at $1077 \mathrm{~cm}^{-1}$ was estimated to be approximately $6.53 \times 10^{7}$.

The proposed PEI-mediated seed growth method can be generally used to synthesize various Ag-coated silica core-shell NPs, ranging from nanoscale to microscale levels. Fig. 5a-c show the TEM images of $\mathrm{SiO}_{2}$ NPs with different sizes (120-300 $\mathrm{nm}$ ), whereas Fig. 5d-f and Fig. 5g-i show their corresponding fabricated $\mathrm{SiO}_{2} @$ PEI-Au seed and $\mathrm{SiO}_{2} @ \mathrm{PEI} @ A g$ NPs, respectively. The obtained $\mathrm{SiO}_{2} @ P E I @ A g$ NPs with different sizes possess good dispersity, uniform $\mathrm{Ag}$ shells, and highly reproducible structure; these particles can be used for different purposes. For example, large-size $\mathrm{SiO}_{2} @ P E I @ A g$ NPs (300-500 $\mathrm{nm}$ ) can act as high-performance SERS substrates, and small NPs $(60-120 \mathrm{~nm})$ can be used as effective SERS tags.

We selected large $\mathrm{SiO}_{2} @$ PEI@Ag NPs $\left(\mathrm{SiO}_{2} 300 \mathrm{~nm}, \mathrm{Ag}\right.$ shell $50 \mathrm{~nm}$ ) as SERS substrate because these particles are large enough to be completely separated by centrifugation and provide sufficient enhancement for direct detection. As illustrated in Scheme 1a, the target analyte with specific affinity could be enriched with $\mathrm{SiO}_{2} @ P E I @ A g$ NPs; the formed complexes were separated by centrifugation and dropped on a silicon chip for SERS measurement. We detected the Raman signals of trace thiram, a widely used sulfur-containing fungicide, ${ }^{37,38}$ to prove the practicability of the $\mathrm{SiO}_{2} @ P E I @ A g$ NPs. The Raman mapping of the thiram at the $1383 \mathrm{~cm}^{-1}$ band confirms the good reproducibility and reliability of Raman detection with $\mathrm{SiO}_{2} @ P E I @ A g$ NPs as SERS substrate (Fig. 6a). Fig. 6b shows the SEM image of the $\mathrm{SiO}_{2} @ P E I @ A g$ NPs covered Si chips from the square area of the 2D-Raman map. Fig. $6 \mathrm{c}$ shows the SERS spectra of thiram in ethanol, with concentrations ranging from $1.0 \times 10^{-5} \mathrm{M}$ to $1.0 \times 10^{-10} \mathrm{M}$ with $\mathrm{SiO}_{2} @ P E I @ A g$ NPs (300 nm core) as SERS substrate; the intensity of the Raman peaks of thiram located at 932, 1148, 1383 , and $1508 \mathrm{~cm}^{-1}$ were observed at concentrations as low as $10^{-8} \mathrm{M}$. The most prominent peak around $1385 \mathrm{~cm}^{-1}$ was observed at concentrations as low as $10^{-9} \mathrm{M}$. All of the major vibrational modes of thiram observed are consistent with previous reports ${ }^{39}$ as categorized in Table S1. $\dagger$ The detection limit of thiram in the experiment $\left(10^{-9} \mathrm{M}\right)$ is lower than the maximum residue limit of $7 \mathrm{ppm}(0.03 \mathrm{mM})$ in fruit, as prescribed by the U.S. EPA. The detection is based on the main Raman peak $\left(1383 \mathrm{~cm}^{-1}\right)$ and then quantified by its intensity. 
(a)

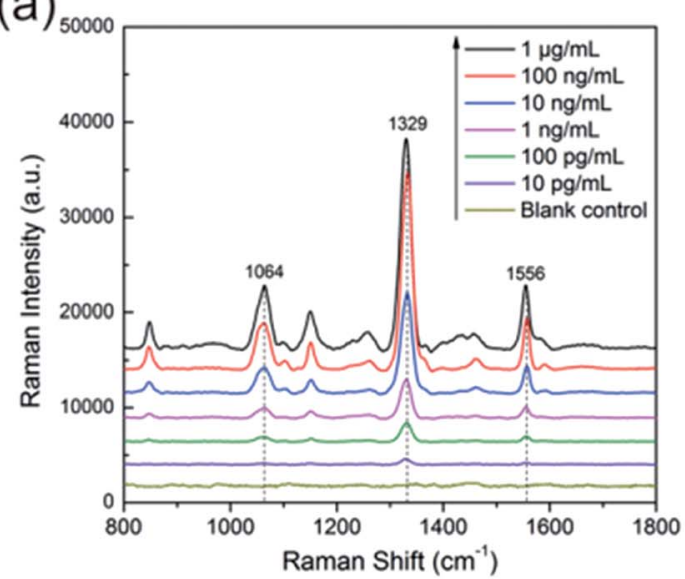

(c)

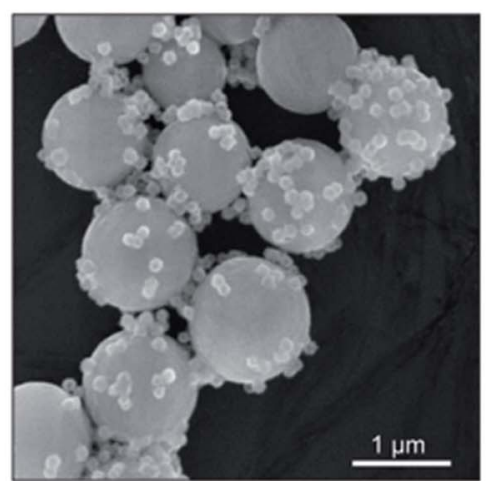

(b)

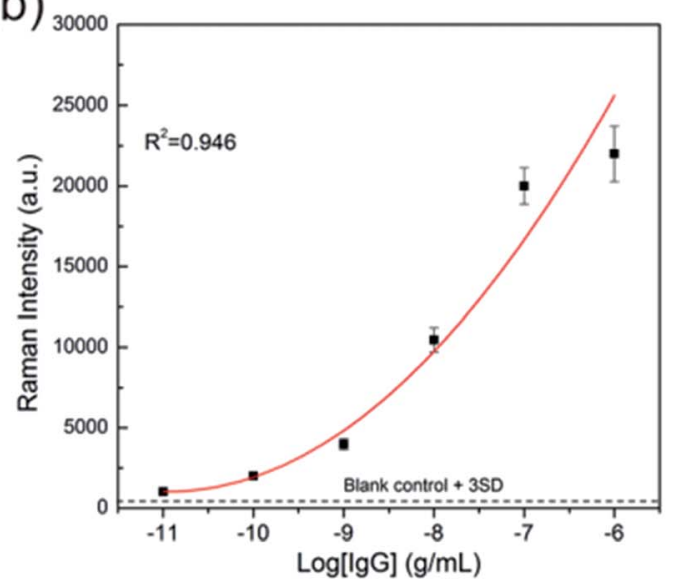

(d)

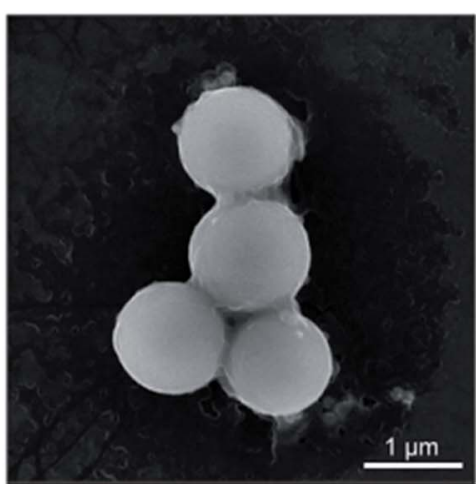

Fig. 7 (a) Concentration-dependent SERS spectra of human IgG ranging from $1 \mu \mathrm{g} \mathrm{mL}^{-1}$ to $10 \mathrm{pg} \mathrm{mL}^{-1}$ and the blank control. (b) Dose-response curve of the above SERS-based immunoassay $\left(R^{2}=0.946\right)$. Error bars indicate the standard deviation obtained from four measurements. SEM images of sandwich immunocomplexes with (c) $1 \mu \mathrm{g} \mathrm{mL} \mathrm{L}^{-1}$ of human $\operatorname{lgG}$ and (d) without human $\lg$.

We conducted five tests for each of the samples and conducted statistical analysis of the results to obtain an intensityconcentration calibration curve (polynomial fitting), which plots the intensity of the SERS peak at $1383 \mathrm{~cm}^{-1}$ versus the logarithmic concentration of the sample (Fig. 6d).

Small $\mathrm{SiO}_{2} @ P E I @ A g$ NPs (60-100 nm) combine the advantages of high SERS activity of $\mathrm{Ag}$ and the homogeneous superiority of $\mathrm{SiO}_{2}$ NPs and show greater surface roughness compared with $\mathrm{Au} / \mathrm{Ag}$ spherical particles of similar size. These NPs can be used as efficient SERS tags for highly reproducible SERS immunoassays. Scheme $1 \mathrm{~b}$ represents the operating principle of the SERS immunoassays using $\mathrm{SiO}_{2} @ P E I @ A g$ SERS tags and immunomagnetic beads, and human IgG is selected as the model target biomolecule to explore the sensitivity of the presented immunoassay protocol. A sandwich coupled plasmonic nanostructure of immunomagnetic beads/IgG/SiO ${ }_{2}$ @PEI@Ag SERS tags was used for human IgG detection. The capture antibody-coated immunomagnetic beads were mainly utilized to magnetically separate and enrich the human IgG in the solution. Impurities were removed, and the detection antibody-coated $\mathrm{SiO}_{2} @ \mathrm{PEI} @ A g$ SERS tags were incubated. These procedures led to the formation of sandwich immune complexes according to immunity affinity. Finally, the formed sandwich immune complexes could be collected quickly and compressed into one dense dot by magnetic attraction, and analyzed with the Raman spectrometer.

$\mathrm{SiO}_{2} @$ PEI@Ag (80 nm core, $10 \mathrm{~nm} \mathrm{Ag} \mathrm{shell)} \mathrm{was} \mathrm{utilized} \mathrm{in}$ the design of efficient SERS tags for SERS immunoassay by labeling DTNB molecules and further coupling detection antibodies via the amidation reaction between the amino groups and preactivated carboxyl groups by EDC and NHS. The micronsized immunomagnetic beads were obtained by a three step synthetic route. The a- $\mathrm{Fe}_{2} \mathrm{O}_{3}$ particles (diameter of $\sim 1.5 \mu \mathrm{m}$ ) possess superparamagnetic property was synthesized first (Fig. S5a $\dagger$ ). Well-dispersed $\mathrm{a}-\mathrm{Fe}_{2} \mathrm{O}_{3} @ \mathrm{SiO}_{2}$ microspheres with a silica shell thickness of $50 \mathrm{~nm}$ were obtained by using Stöber method..$^{40}$ The transparent edge of the core-shell microstructure in Fig. S5b† implies the successful coating of silica around the magnetic core. Finally, the $\mathrm{a}-\mathrm{Fe}_{2} \mathrm{O}_{3} @ \mathrm{SiO}_{2}$ microspheres were carboxyl-modified with TEPSA; the capture antibodies were conjugated on the magnetic microspheres by covalent bonding via the EDC/NHS chemistry.

Fig. 7a Illustrates the SERS spectra of the sandwich immunocomplex for various concentrations of human IgG. The concentration of human IgG was varied from $10 \mathrm{pg} \mathrm{mL}^{-1}$ to $1 \mu \mathrm{g}$ $\mathrm{mL}^{-1}$. Each spectrum is the average of five SERS spectra and has undergone baseline subtraction. The power at the samples was $1 \%$ of the full laser power and the integration time was $1 \mathrm{~s}$. The 
Raman peak of DTNB centered at $1329 \mathrm{~cm}^{-1}$ (symmetric $\mathrm{NO}_{2}$ stretching bands) was used for quantitative evaluation of human IgG. The calibration curve is shown in Fig. 7b, where the error bars indicate standard deviations from four measurements. The limit of detection (LOD) is defined as the analyte concentration that produces a signal three times larger than the standard deviation of the blank control ${ }^{\mathbf{4 1}}$ hence, the LOD of the immunoassay protocol was $10 \mathrm{pg} \mathrm{mL}^{-1}$. The SEM images demonstrated that the SERS signal of the immunocomplex originated from the $\mathrm{SiO}_{2} @ P E I @ A g$ SERS tags linked to the surface of the immunomagnetic beads. As shown in Fig. 7c, a large number of $\mathrm{SiO}_{2}$ @PEI@Ag SERS tags (80 nm core) were coupled with immunomagnetic beads because of immunity affinity in the presence of the target human IgG, whereas the blank control experiment indicated no binding of the SERS tags to immunomagnetic beads (Fig. 7d). Basing on the results, we demonstrated the great potential of these $\mathrm{SiO}_{2} @ P E I @ A g$ NPs (80 $\mathrm{nm}$ core) as effective SERS tag for highly selective and sensitive protein detection.

\section{Conclusions}

We demonstrate a facile and effective polyethyleneiminemediated seed growth method for fabrication of novel $\mathrm{SiO}_{2} @$ PEI@Ag NPs with monodispersity, high homogeneity, and a complete Ag shell. In these NPs, PEI was skillfully used to absorb dense Au seeds and prevent particle aggregation. The coverage lever and thickness of the $\mathrm{Ag}$ nanoshell can be easily tailored by varying the concentration of $\mathrm{AgNO}_{3}$. The detailed nanostructures of the $\mathrm{SiO}_{2} @ \mathrm{PEI} @ A g$ NPs were characterized by TEM, SEM, XRD, X-ray elemental mapping technique, and UVvisible spectroscopy. To the best of our knowledge, this study is the first to report the use of polymer PEI to grow complete Ag shells with controllable thickness on silica spheres. Moreover, the newly developed PEI-mediated seed growth method can be generally used to synthesize various $\mathrm{SiO}_{2} @ P E I @ A g ~ N P s$, ranging from nanoscale to microscale. These obtained $\mathrm{SiO}_{2} @$ PEI@Ag NPs with different sizes could produce highly enhanced Raman signals with good uniformity and reproducibility because of its uniform Ag shells and highly reproducible structure, which can be used as versatile SERS platforms for different purposes. We further demonstrated that large $\mathrm{SiO}_{2}$ @PEI@Ag NPs (300 nm core) can be used as effective SERS substrates, as verified by the detection of pesticide thiram with a detection limit of $10^{-9}$ M. Small SiO @PEI@Ag NPs (80 nm core) as high-quality SERS tags were successfully applied to SERS-based immunoassay for human IgG detection, with detection limit as low as $10 \mathrm{pg} \mathrm{mL^{-1 } \text { . Therefore, } \mathrm { SiO } _ { 2 } @ P E I @ A g}$ NPs can be used as an excellent SERS platform for chemical and biological analyses.

\section{Acknowledgements}

This work was supported by Grants from the National Natural Science Foundation of China (No. 81230089 and 51605486), and Beijing Municipal Science \& Technology Commission (No. Z161100000116040).

\section{Notes and references}

1 T. Ung, L. M. Liz-Marzán and P. Mulvaney, J. Phys. Chem. B, 1999, 103, 6770-6773.

2 M. Lv, S. Su, Y. He, Q. Huang, W. Hu, D. Li, C. Fan and S. T. Lee, Adv. Mater., 2010, 22, 5463-5467.

3 J.-B. Zeng, Y.-Y. Cao, J.-J. Chen, X.-D. Wang, J.-F. Yu, B.-B. Yu, Z.-F. Yan and X. Chen, Nanoscale, 2014, 6, 9939-9943.

4 B. Rodríguez-González, F. Attouchi, M. F. Cardinal, V. Myroshnychenko, O. Stéphan, F. J. García de Abajo, L. M. Liz-Marzán and M. Kociak, Langmuir, 2012, 28, 90639070.

5 A. Sánchez-Iglesias, P. Aldeanueva-Potel, W. Ni, J. PérezJuste, I. Pastoriza-Santos, R. A. Alvarez-Puebla, B. N. Mbenkum and L. M. Liz-Marzán, Nano Today, 2010, 5, 21-27.

6 C. Y. Li, M. Meng, S. C. Huang, L. Li, S. R. Huang, S. Chen, L. Y. Meng, R. Panneerselvam, S. J. Zhang, B. Ren, Z. L. Yang, J. F. Li and Z. Q. Tian, J. Am. Chem. Soc., 2015, 137, 13784-13787.

7 K. Wang, X. Zhang, C. Niu and Y. Wang, ACS Appl. Mater. Interfaces, 2014, 6, 1272-1278.

8 J. K. Yang, H. Kang, H. Lee, A. Jo, S. Jeong, S. J. Jeon, H. I. Kim, H. Y. Lee, D. H. Jeong, J. H. Kim and Y. S. Lee, ACS Appl. Mater. Interfaces, 2014, 6, 12541-12549.

9 J. B. Liu, W. Dong, P. Zhan, S. Z. Wang, J. H. Zhang and Z. L. Wang, Langmuir, 2005, 21, 1683-1686.

10 T.-H. Chen, R.-D. Jean, K.-C. Chiu, C.-H. Chen and D.-M. Liu, Appl. Phys. Lett., 2012, 100, 223101.

11 K. Kim, H. B. Lee, Y. M. Lee and K. S. Shin, Biosens. Bioelectron., 2009, 24, 1864-1869.

12 T. Liu, D. Li, D. Yang and M. Jiang, Colloids Surf., A, 2011, 387, 17-22.

13 Y. Kobayashi, V. Salgueiriño-Maceira and L. M. Liz-Marzán, Chem. Mater., 2001, 13, 1630-1633.

14 G. Ren, W. Wang, M. Shang, H. Zou and S. Cheng, Langmuir, 2015, 31, 10517-10523.

15 K. Kim, H. S. Kim and H. K. Park, Langmuir, 2006, 22, 80838088.

16 Z.-J. Jiang and C.-Y. Liu, J. Phys. Chem. B, 2003, 107, 1241112415.

17 R. Wang, X. Ji, Z. Huang, Y. Xue, D. Wang and W. Yang, J. Phys. Chem. C, 2016, 120, 377-385.

18 L. Gomez, M. Arruebo, V. Sebastian, L. Gutierrez and J. Santamaria, J. Mater. Chem., 2012, 22, 21420-21425.

19 C. Wang, J. Xu, J. Wang, Z. Rong, P. Li, R. Xiao and S. Wang, J. Mater. Chem. C, 2015, 3, 8684-8693.

20 C. Wang, P. Li, J. Wang, Z. Rong, Y. Pang, J. Xu, P. Dong, R. Xiao and S. Wang, Nanoscale, 2015, 7, 18694-18707.

21 J. Wang, X. Wu, C. Wang, Z. Rong, H. Ding, H. Li, S. Li, N. Shao, P. Dong, R. Xiao and S. Wang, ACS Appl. Mater. Interfaces, 2016, 8, 19958-19967.

22 P. Guo, D. Sikdar, X. Huang, K. J. Si, W. Xiong, S. Gong, L. W. Yap, M. Premaratne and W. Cheng, Nanoscale, 2015, 7, 2862-2868. 
23 Y. Fang, S. Guo, C. Zhu, Y. Zhai and E. Wang, Langmuir, 2010, 26, 11277-11282.

24 Z. Gan, A. Zhao, Q. Gao, M. Zhang, D. Wang, H. Guo, W. Tao, D. Li, E. Liu and R. Mao, RSC Adv., 2012, 2, 8681.

25 M. Li, J. Zhang, S. Suri, L. J. Sooter, D. Ma and N. Wu, Anal. Chem., 2012, 84, 2837-2842.

26 M. Li, J. W. Kang, S. Sukumar, R. R. Dasari and I. Barman, Chem. Sci., 2015, 6, 3906-3914.

27 T. Liu, D. Li, D. Yang and M. Jiang, Chem. Commun., 2011, 47, 5169-5171.

28 Q. An, P. Zhang, J. M. Li, W. F. Ma, J. Guo, J. Hu and C. C. Wang, Nanoscale, 2012, 4, 5210-5216.

29 F. Ke, L. Wang and J. Zhu, Nanoscale, 2015, 7, 1201-1208.

30 J. Ma, K. Wang and M. Zhan, ACS Appl. Mater. Interfaces, 2015, 7, 16027-16039.

31 Q. An, M. Yu, Y. Zhang, W. Ma, J. Guo and C. Wang, J. Phys. Chem. C, 2012, 116, 22432-22440.

32 N. Gao, T. Yang, T. Liu, Y. Zou and J. Jiang, RSC Adv., 2015, 5, 55801-55807.
33 J. F. Li, X. D. Tian, S. B. Li, J. R. Anema, Z. L. Yang, Y. Ding, Y. F. Wu, Y. M. Zeng, Q. Z. Chen, B. Ren, Z. L. Wang and Z. Q. Tian, Nat. Protoc., 2013, 8, 52-65.

34 Y. Zhao, Y.-J. Zhang, J.-H. Meng, S. Chen, R. Panneerselvam, C.-Y. Li, S. B. Jamali, X. Li, Z.-L. Yang, J.-F. Li and Z.-Q. Tian, J. Raman Spectrosc., 2016, 47, 662-667.

35 D.-Y. Wu, X.-M. Liu, Y.-F. Huang, B. Ren, X. Xu and Z.-Q. Tian, J. Phys. Chem. C, 2009, 113, 18212-18222.

36 Y. F. Huang, D. Y. Wu, H. P. Zhu, L. B. Zhao, G. K. Liu, B. Ren and Z. Q. Tian, PCCP Phys. Chem. Chem. Phys., 2012, 14, 8485-8497.

37 S. Liu, C. Jiang, B. Yang, Z. Zhang and M. Han, RSC Adv., 2014, 4, 42358-42363.

38 X. Zhang, C. Niu, Y. Wang, S. Zhou and J. Liu, Nanoscale, 2014, 6, 12618-12625.

39 C. Niu, B. Zou, Y. Wang, L. Cheng, H. Zheng and S. Zhou, Langmuir, 2016, 32, 858-863.

40 Y. Wang, K. Wang, B. Zou, T. Gao, X. Zhang, Z. Du and S. Zhou, J. Mater. Chem. C, 2013, 1, 2441.

41 B. Zhao, J. Shen, S. Chen, D. Wang, F. Li, S. Mathur, S. Song and C. Fan, Chem. Sci., 2014, 5, 4460-4466. 